\title{
Journal of Clinical and Translational Science
}

\section{Diagnostic accuracy of damage-associated molecular patterns (DAMPs) in patients with heart failure with a reduced ejection fraction}

\author{
Justin Hartupee, Rohan Patel, Lora Staloch, Victor G. Dávila-Román, \\ Lisa de las Fuentes, Eric Novak and Douglas L. Mann*
}

Department of Medicine, Center for Cardiovascular Research, Cardiovascular Division, Washington University School of Medicine, St. Louis, MO, USA

Journal of Clinical and Translational Science (2017), I, pp. 208-209 doi:10.1017/cts.2017.II

To the Editor

Elevated levels of inflammatory mediators have been identified in patients with heart failure (HF) with a reduced ejection fraction (HFrEF) in direct relation to worsening New York Heart Association (NYHA) class [I]. However, the mechanism(s) responsible for elevated levels of inflammatory mediators in HF is not known. Recent studies have shown that dying cells release "damage-associated molecular patterns" (DAMPs) that provoke inflammatory responses through engagement of innate immune receptors (e.g., toll-like receptor 4) [2]. Accordingly we sought to determine whether DAMPs were elevated in HF patients in relation to NYHA class, as well as assess their diagnostic accuracy for HF. We examined the DAMP galectin-3 [3] which has a US Food and Drug Administration-approved assay for the diagnosis of HF, as well as two other DAMPs that have been linked to inflammation: calprotectin [SI00A8/SI00A9] and high mobility box group box I (HMGBI). NT-proBNP was used as the reference standard for diagnosing $\mathrm{HF}$, and circulating levels of troponin T were used as a sensitive marker of myocyte injury in HF.

We measured circulating levels of the biomarkers in 120 controls (EF $>55 \%$ ) and 120 cases with NYHA class I-IV HFrEF (EF $<40 \%)$ from the Washington University Heart Failure registry [4] who were matched on age, gender, diabetes, and hypertension. Measurement of NT-proBNP and high-sensitivity (hs) troponin T were measured using a commercial assay (Roche Diagnostics) and galectin-3 (R\&D Systems), calprotectin (Hycult Biotech), and HMGBI (IBL International) were measured by ELISA.

Fig. I shows that circulating levels of NT-proBNP (a), hs-troponin T (b), and galectin-3 (c) increased with worsening NYHA class; whereas levels

* Address for correspondence: D. L. Mann, M.D., Division of Cardiology, Washington University School of Medicine, 660S. Euclid Ave, Campus Box 8086, St. Louis, MO 63I I0, USA. (Email: dmann@wustl.edu) of HMGBI (d) were elevated similarly in NYHA class II-IV HF and levels of calprotectin (e) were only elevated in NYHA class IV HFrEF. We next assessed Receiver Operating Characteristics curves for each biomarker to determine their accuracy to discriminate HFrEF from controls (f). As shown in Table I, the rank order for diagnostic accuracy was NT-proBNP > hs-troponin T > galectin-3 > calprotectin > HMGBI. The diagnostic accuracy of NT-proBNP was significantly better than any of the biomarkers studied [5]. Interesting, neither calprotectin nor HMGBI were accurate in terms of diagnosing patients with HFrEF.

We conclude that although classical DAMPs such as HMGBI and calprotectin are elevated in HFrEF patients, they do not track with NYHA class, and therefore are not likely to explain the increased levels of inflammatory mediators observed in HF, nor are these DAMPs as accurate for diagnosing HF as traditional markers of cell injury, such as troponin $\mathrm{T}$.

\section{Acknowledgments}

This study was supported by the Mentors in Medicine program in the Department of Medicine at Washington University, Roche Diagnostics (NT-proBNP and hs-Troponin T assays) and NIH RC2-HLI02222.

\section{References}

I. Mann DL. Innate immunity and the failing heart: the cytokine hypothesis revisited. Circulation Research 2015; I 16: 1254-1268.

2. Mann DL. The emerging role of innate immunity in the heart and vascular system: for whom the cell tolls. Circulation Research 201 I; 108 : I |33-I | 45.

3. Sato S, et al. Galectins in innate immunity: dual functions of host soluble beta-galactoside-binding lectins as damage-associated molecular patterns (DAMPs) and as receptors for pathogen-associated molecular patterns (PAMPs). Immunological Reviews 2009; 230: 172-187. 
(a)

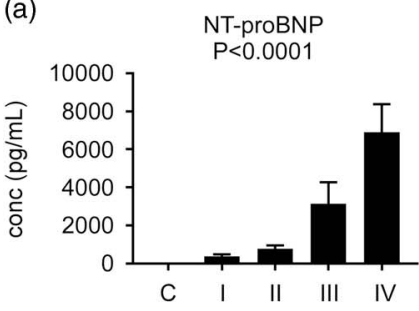

(b)

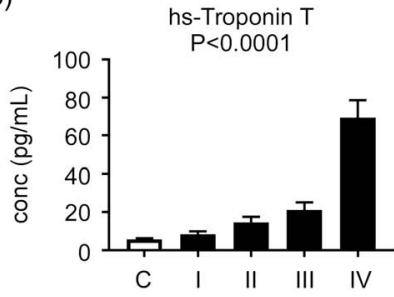

(c)

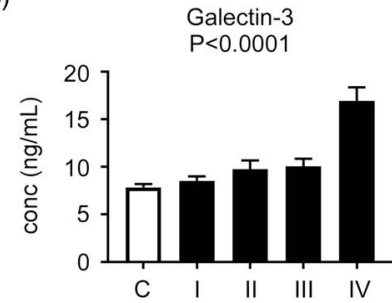

(d)

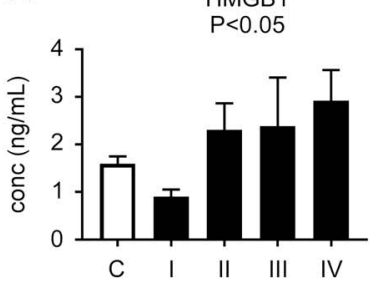

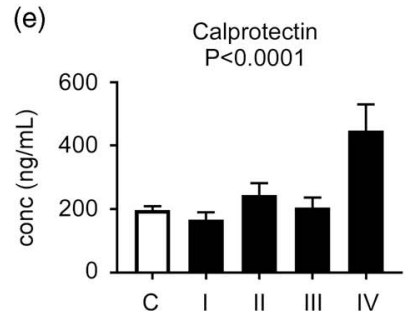

(f)

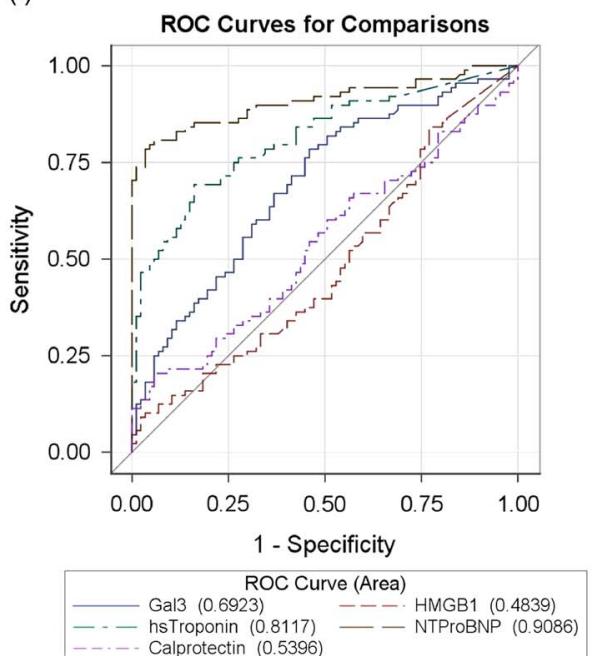

Fig. I. Damage-associated molecular patterns in heart failure (HF) with reduced ejection fraction (EF). (a-e) Biomarker levels in controls (C) and patients with New York Heart Association class I-IV HF for NT-proBNP (a), high-sensitivity (hs) troponin (b), galectin-3 (c), HMGBI (d), and calprotectin (e). Data expressed as mean and error bars represent SEM. $p$ Values determined by I-way analysis of variance. ( $f$ ) The ability of different biomarkers to discriminate HF from controls was evaluated by determining the area under the Receiver Operating Characteristic (ROC) curve. The area under the curve ranges from 0 to I and describes accuracy, with I identifying perfect predictive ability and 0.5 indicating that performance is no better than chance.

Table I. Diagnostic accuracy of biomakers assessing area under curve (AUC)

\begin{tabular}{lcccc}
\hline Predictor & AUC & $95 \% \mathrm{Cl}$ & $p$-value & $p$-value vs. NT-proBNP \\
\hline NT-proBNP & $0.9 \mathrm{I}$ & $(0.86,0.96)$ & $<0.00 \mathrm{I}$ & - \\
hs-Troponin T & $0.8 \mathrm{I}$ & $(0.75,0.88)$ & $<0.00 \mathrm{I}$ & 0.002 \\
Galectin-3 & 0.69 & $(0.6 \mathrm{I}, 0.77)$ & $<0.00 \mathrm{I}$ & $<0.00 \mathrm{I}$ \\
Calprotectin & 0.54 & $(0.45,0.63)$ & 0.37 & $<0.00 \mathrm{I}$ \\
HMGBI & 0.48 & $(0.40,0.57)$ & $0.7 \mathrm{I}$ & $<0.00 \mathrm{I}$ \\
\end{tabular}

$\mathrm{Cl}$, confidence intervals; HMGBI, high mobility group box I; hs, high sensitivity.
4. Joseph SM, et al. Comparable performance of the Kansas City Cardiomyopathy Questionnaire in heart failure patients with preserved and reduced ejection fraction. Circulation. Heart Failure 2013; 6: | |39-| | 46.

5. DeLong ER, DeLong DM, Clarke-Pearson DL. Comparing the areas under two or more correlated receiver operating characteristic curves: a nonparametric approach. Biometrics 1988; 44: 837-845. 